\title{
Various Neurological Manifestations of Gestational Hypertension
}

\author{
Author.Dr K.Murugalakshmi MD,DGO \\ Co Author Dr.V.Nandhini DGO,DNB(OG) \\ Author: Associate Professor, Co Author: Assistant professor \\ Department of obstetrics\& gynecology Coimbatore Medical College Hospital, Coimbatore, Tamilnadu
}

\begin{abstract}
Aim And Objectives Of The Study: 1. To find the correlation of various indices of gestational hypertension with neurological manifestations, like gestational age of onset of signs and symptoms, systolic blood pressure ,recurrence of gestational hypertension, weight gain during pregnancy, family history, co morbid conditions like anaemia, gestational diabetes mellitus etc,.

2. Early identification using related indicators and treat them accordingly to prevent the catastrophic neurological diseases and their sequelae.

Keywords: Preeclampsia, eclampsia, cvt.
\end{abstract}

\section{Introduction}

Gestational hypertension occurs in $25-30 \%$ of pregnant population in the developing countries and it forms one of the triad of direct causes of maternal mortality. It is also associated with long term risk for hypertension, stroke and heart disease. Cerebrovascular complication occur in only a small portion of women with severe preeclampsia but with high morbidity and mortality. Endothelial dysfunction and impaired cerebral autoregulation and severe hypertension in the setting of preeclampsia are the causes for mortality and morbidity. The purpose of this review is to describe the epidemiology of preeclampsia and related risk factors for the neurological disease.

\section{Materials And Methods}

Observational study done in the patients who are diagnosed and labelled as gestational hypertensives in the department of obstetrics and gynecology ,Coimbatore medical college hospital ,Coimbatore, during the month of April,May of 2016.

Spectrum of gestational hypertension

\section{Review Of Literature}

Gestational hypertension: Preeclampsia is a multisystem disorder of pregnancy which is characterised by new onset hypertension ( systolic and diastolic blood pressure of $>140$ and 90mm hg,on two occasions atleast six hours apart ).,that develop after 20 weeks of gestation in previously normotensive women .

Preeclampsia :Gestational hypertension with proteinuria( protein excretion of $>300 \mathrm{mg}$ in 24 hours urine collection or dipstick of $>2+$ )

Preeclampsia early onset: Starting before 34 weeks of gestation

Preeclampsia late onset: Starting after 34 weeks of gestation

Mild preeclampsia: $\mathrm{BP}<160 / 110$

Severe preeclampsia: $\mathrm{BP}>160 / 110$, proteinuria $>5 \mathrm{gms} / 24 \mathrm{hrs}$, oliguria, HELLP syndrome

Imminent eclampsia: Severe preeclampsia with symptoms like vomiting,headache,epigastric pain , visual disturbances.

Eclampsia: Preeclampsia associated with seizures

Postpartum headache: Preeclampsia is often associated with syndrome of reversible posterior leukoencephalopathy syndrome (RPLS, also known as PRES)it is usually due to reversible vasogenic edema as seen on CT/MRI usualy in the occipital or parietal lobes. Reversible cerebral vasoconstriction syndrome (RCVS) also known as Call Fleming syndrome has an insidious onset typically called as thunderclap headache.

CVT : Due to cortico venous thrombosis or hemorrhage, the patient may present with stroke like syndrome.

\section{Pathogenesis Of Neurologic Complications Of Preeclampsia.}

Two theories are postulated to explain cerebral abnormalities associated with Eclampsia. Endothelial dysfunctions play a central role in both theories. The $1^{\mathrm{ST}}$ theory suggest that vasospasm of cerebral vasculature in women with severe preeclampsia and Eclampsia is a response to acute severe hypertension (cerebral overregulation leads to vasospasm). Vasospasm results in cytotoxic oedema, ischemia and tissue infarction. Alternate theory suggests that sudden elevation in systemic blood pressure may exceed cerebral vascular auto 
regulation, regions of vasoconstriction and forced vasodilatation develop resulting in increased hydrostatic pressure and extravasation of plasma and RBC and vasogenic oedema described as posterior reversible leucoencephalopathy syndrome(PRES).

\section{Cerebral Hemodynamics In Pregnancy And Preeclampsia.}

Transcranial Doppler ultrasound shows increased cerebral blood flow velocity in preeclampsia at term compared with normotensive pregnant women. The increase in cerebral blood flow velocity is due to change in resistance of arterioles, increased cardiac output, increase in mean arterial blood pressure .The increase in cerebral blood flow contributes to cerebrovascular auto regulatory breakthrough in PRES preeclampsia and Eclampsia.

\section{Hemorrhagic Complication}

Sudden death occurs at the time of seizures or shortly thereafter is a result of massive cerebral haemorrhage. The cause of such haemorrhage is long standing hypertension induced lipohyalinosis which damages small or medium sized arteries, common in older women underlying hypertension. The striatocapsular area, Thalamus, Cerebellum, brainstem are the sites commonly affected. Alternatively cerebral infarction may transform into haemarrghic infarction, such happen in young nullipara who present with HELLP syndrome and Eclampsia. Hypertension and coagulopathy increase the risk of haemorrhagic transformation into previously ischemic areas of brain. Subarachnoid haemorrhage is occasionally reported and is due to rupture of cortical petechiae over the brain surface or rupture of small pial veins, carries benign prognosis.

\section{Visual Disturbances}

Visual disturbances like scotoma, amaurosis, blurred vision ,diplopia may occur in $40 \%$ of preeclamptic female. Neuro imaging modalities reveal focal cerebral oedema, including bilateral oedema of lateral geniculate nuclei, therefore the term" cortical blindness" was introduced. The lesions are seen particularly in the parieto-occipital area and supplied by circulation and are reversible.

\section{Seizures}

Most seizure occur during intrapartum and postpartum period. Symptom may occur before or after onset of convulsions and include persistent occipital headache, blurred vision, photophobia, epigastric or right upper quadrant pain and altered mental status. In $60-75 \%$ of cases one of these symptoms is seen. The most consistent prodrom of seizures is severe throbbing headache, which herald the loss of auto regulation.

Eclampsia And Brain: remote prognosis.

Almost 1/4 eclamptic women demonstrate white matter lesions 6-8 week postpartum. Recent data demonstrate long term persistence of brain matter lesion possibly incurred at the time of eclamptic convulsion. Cerebrovascular events in Eclampsia appear to constitute a continuum characterised by an initial reversible phase of vasogenic oedema caused by hypertension along with endothelial dysfunction i some cases vasogenic oedema ma y reduce cerebral perfusion to cause focal ischemia. The high incidence of persistent brainstem lesion in formerly eclamptic female raises the possibility that they develop a small degree of subtle brain dysfunction. This would contradict the predominant conceptualization of Eclampsia as a condition of which female can expect full clinical recovery.

\section{Observation}

1. Out of 250 patients observed who presented as gestational hypertension 50, remained so without progressing to complication, 200 turned out to be proteinuric and hence labelled as preeclamptic. Of them 100 had neurological complications and 100 were uncomplicated preeclamptic .

Table 1

\begin{tabular}{|l|l|l|}
\hline SPECTRUM OFGHT & NUMBER & PERCENTAGE \\
\hline Remained as GHT & $50 / 250$ & $20 \%$ \\
\hline Preeclampsia & $200 / 250$ & $80 \%$ \\
\hline Preeclampsia \& neurologic complication & $100 / 250$ & $40 \%$ \\
\hline
\end{tabular}

Table 2 Spectrum Of Neurological Manifestations

\begin{tabular}{|l|l|l|}
\hline Spectrum & Number & Percentage \\
\hline Imminent Eclampsia & 68 & $68 \%$ \\
\hline Eclampsia & 22 & $22 \%$ \\
\hline Postpartum Headache & 5 & $5 \%$ \\
\hline Cvt & 5 & $5 \%$ \\
\hline
\end{tabular}


2.Anaemia is a comorbid condition associated with Preeclampsia and those patients with neurological complication have still more association with anaemia.

Table 3

\begin{tabular}{|l|l|l|}
\hline Anaemia \& GHT & $40 / 250$ & $16 \%$ \\
\hline Anaemia\& PREECLAMPSIA & $28 / 200$ & $14 \%$ \\
\hline Anaemia \& neurological complication & $21 / 100$ & $21 \%$ \\
\hline
\end{tabular}

Anaemia adds to the fuel by increasing the incidence more than $20 \%$.

3.Preeclampsia have an increased incidence of perinatal morbidity \& mortality due to LBW(early termination )\&IUGR. It has been observed that there is an increased association of adverse perinatal outcome with those hypertensives with neurological complications.

Table 4

\begin{tabular}{|l|l|l|}
\hline & Gestational hypertensives & With neurologic complication \\
\hline IUGR & $81 / 250$ & $34 / 100$ \\
\hline -LBW & $27 / 250$ & $7 / 100$ \\
\hline IUFD & $17 / 250$ & $14 / 100$ \\
\hline
\end{tabular}

The cause being the same for both preeclampsia and IUGR/LBW there is increased association with adverse perinatal outcome.

4As per literature there is increased incidence of neurological complication, if there is early onset $(<34$ weeks) of GHT. Comparing those with early onset and late onset the spectrum varies.

Table 5

\begin{tabular}{|l|l|l|l|l|}
\hline SPECTRUM & Early onset(70) & $\%$ & Late onset(180) & $\%$ \\
\hline GHT & 12 & 17 & 38 & 21 \\
\hline Preeclampsia & 18 & 26 & 82 & 46 \\
\hline Imminent eclampsia & 22 & 32 & 46 & 25 \\
\hline Eclampsia & 12 & 17 & 10 & 6 \\
\hline Postpartum headache & 3 & 4 & 2 & 1 \\
\hline CVT & 3 & 4 & 2 & 1 \\
\hline
\end{tabular}

Neurological complications are more in early onset GHT 57\% compared with late onset -33\%.Combining both early onset GHT have increased incidence of perinatal morbidity and mortality.

Table 6

\begin{tabular}{|l|l|l|l|l|}
\hline SPECTRUM & Early onset(70) & $\%$ & Late onset(180) & $\%$ \\
\hline IUGR & $26 / 70$ & 37 & $55 / 180$ & 30 \\
\hline LBW & $9 / 70$ & 13 & $18 / 180$ & 10 \\
\hline IUFD & $10 / 170$ & 14 & $7 / 180$ & 3 \\
\hline
\end{tabular}

Out of the 70 early onset cases 45 i.e $64 \%$ are associated with adverse perinatal out come but out of the 180 late onset cases 85 i.e $47 \%$ are associated with perinatal out come

Early onset GHT have increased association with other comorbid condition like anaemia, GDM HYPOTHYROIDISM and recurrence rate is also high and family h/o is also positive in many cases.

5. Usually it has been taught that Diastolic BP determines the adverse outcome $s$ and hence treatment is more concentrated towards lowering the diastolic BP,but this study has revealed systolic $\mathrm{BP}(>160)$ has more neurological problem than diastolic BP $(>110)$.

Table 7

\begin{tabular}{|l|l|l|l|l|l|l|l|}
\hline Systolic BP & $<140$ & 150 & $>160$ & Diastolic BP & $<90$ & $90-109$ & $>110$ \\
\hline$\%$ & 74 & 66 & 110 & ---- & 106 & 123 & 18 \\
\hline $\begin{array}{l}\text { Associated } \\
\text { neurologic } \\
\text { symptom }\end{array}$ & 17 & 37 & 46 & --- & 27 & 60 & 13 \\
\hline
\end{tabular}

Out of the 100 patients who had neurological complications $46 \%$ have a systolic Bp>160 but only $13 \%$ have diastolic $\mathrm{Bp}>110$

6. Family history also plays a vital role in determining the incidence of gestational hypertension and its complications. Out of 250 cases 68 cases i.e., approximately $1 / 4$ of cases have a positive family history either 
mother HT/ Father HT or CVA or IHD. But there is a strong correlation between mother being hypertensive, CVA or IHD.

Table 8

\begin{tabular}{|l|l|l|l|}
\hline & HT & CVA & IHD \\
\hline Mother & 42 & 1 & 1 \\
\hline Father & 17 & 4 & 3 \\
\hline
\end{tabular}

Out of 68 cases 31 had neurologic complications. This shows family history is a major risk factor. Out of 68 cases 14 have an early onset and hence the complications.

7. Considering the weight gain during the pregnancy only 21 cases have got an increase of $>15 \mathrm{~kg}$ but there were also associated with co morbid conditions like GDM and Hypothyroidism. There were $>144$ cases who have gained $<10 \mathrm{~kg}$, these people are associated with anaemia hence not only overweight but poor weight gain also may be a causative factor( cause being defective angiogenesis)

Table 9

\begin{tabular}{|l|l|l|l|}
\hline & $<10 \mathrm{~kg}$ & $11-15 \mathrm{~kg}$ & $>15 \mathrm{~kg}$ \\
\hline No.of patients & $144 / 250$ & $85 / 250$ & $21 / 250$ \\
\hline Neuro complication & 54 & 34 & 12 \\
\hline
\end{tabular}

\section{Discussion}

Gestational hypertention being a common clinical entity of pregnant population is being influenced by many of the risk factor like weight gain during pregnancy, onset of signs and symptoms, associated comorbid conditions like anemia,GDM,hypothyroidism, recurrency,family history of hypertension leads to both maternal and fetal adverse outcome.Adverse maternal outcome may due to neurological complication, cardiovascular problem or multi organ damage.But neurological complications are usually unpredictive and may leave a permanent damage to the patients .Hence they have to be identified using indices in the early months of pregnancy and get it treated .From the above study it is revealed anaemia if present in a preeclamptic patient she is prone for neurological complications (21/100), if IUGR is detected in a preeclamptic patient incidence is increased (34/100).If the signs and symptoms appear earlier the association is still more stronger (54\% vs 33\%). A high systolic BP $(>160)$ is more influential than diastolic BP $>110$. A family history of HT/CVA/IHD also indicates the severity of disease.

Hence these above said indices may be considered as HIGH RISK factors for neurological complciations and sequelae of Gestational Hypertension.

\section{Conclusion}

The study revealed the association of these high risk factors in a patient with Gestational Hypertension will lead to catastrophic neurological complications. Hence earlier booking and identifying these high risk factors in these patients should be a must to prevent both maternal and perinatal adverse outcomes.

\section{Bibliography}

[1]. Neurological complications of Eclampsia. (PMID:10862315), Sawhney IM, Subrahmanyan AV , Das CP, Khandelwal N, Sawhney H, Vasishta K. Dept. of Neurology, Postgraduate Institute of Medical Education and Research, Chandigarh, India. The Journal of the Association of Physicians of India [1999, 47(11):1068-1071]

[2]. Neurological complications in Eclampsia: a case series. (PMID:15188830)

Tank PD, Chauhan AR, Bhattacharya MS, Warke HS, Raut VS

International journal of fertility and women's medicine [2004 Mar-Apr; 49(2):61-9]

[3]. Neurological aspects of Eclampsia. (PMID:9562320)

Thomas SV , Journal of the neurological sciences [1998 Feb 18;155(1):37-43]

[4]. Idiopathic generalized epilepsies imitating focal epilepsies. (PMID:16302881) Ferrie CD Epilepsia [2005;46 Suppl 9:91-5]

[5]. J Neurol Neurosurg Psychiatry 1988;51:416- 421 doi:10.1136/jnnp.51.3.416 Clinicopathological study of neurological complications due to hypertensive disorders of pregnancy. A Richards, D Graham, R Bullock

[6]. Cerebral vascular adaptation to pregnancy and its role in the neurological complications of Eclampsia. Marilyn J. Cipolla, Julie G. Sweet, SiuLungChan.JournalofAppliedPhysiology Published 1 February 2011 Vol. 110 no. 2, 329-339 DOI: 10.1152/japplphysiol.01159.2010

[7]. Seminars in perinatology. Neurologic Complications of Pre-Eclampsia , Gerda G. Zeeman, MD, June 2009, Volume 33, Issue 3, Pages 166-172

[8]. American Journal of Obstetrics and Gynecology, Volume 152, Issue 2, 15 May 1985, Pages 184-192 Eclampsia: IV. Neurological findings and future outcome. Baha M. Sibai, M.D. a, Joseph A. Spinnato, M.D., Dottie L. Watson, M.D., James A. Lewis, M.D., Garland D. Anderson, M.D. 
[9]. Cerebral imaging in eclampsia: Magnetic resonance imaging versus computed tomography ,Margaret A. Dahmus, MD ' 1, John R. Barton, MD , Baha M. Sibai, MD , . American Journal of Obstetrics and Gynaecology, Volume 167, Issue 4, Part 1, October 1992, Pages 935-941

[10]. Seminars in Perinatology,Volume 37, Issue 4, August 2013, Pages 207-224, Seizures in pregnancy: Epilepsy, eclampsia, and stroke, Laura A. Hart, MD, Baha M. Sibai, MD

[11]. Neurological complications in eclampsia: a case series.(PMID:15188830) Tank PD, Chauhan AR, Bhattacharya MS, Warke S , Raut VS, International Journal of Fertility and Women's Medicine [2004, 49(2):61-69]

[12]. Severe preeclampsia and eclampsia in Kerman, Iran: complications and outcomes. Bibi Shahnaz Aali, Jila Ghafoorian, Sakineh Mohamad-Alizadeh. Med Sci Monit 2004; 10(4): CR163-167

[13]. A comparison of maternal serum levels of endothelial nitric oxide synthase, asymmetric dimethylarginine, and homocysteine in normal and preeclamptic pregnancies. Marzena Laskowska, Katarzyna Laskowska, Mahfoz Terbosh, Jan Oleszczuk. Med Sci Monit 2013; 19:430-437 :: DOI: 10.12659/MSM.883932.Published: 2013-06-05

[14]. The American Journal of Emergency Medicine,Volume 21, Issue 3, May 2003, Pages $223-226$ A current concept of Eclampsia.Hadassah Lipstein, MD $^{\mathrm{a}}$, Christopher C Lee, MD ${ }^{\mathrm{a},}$, Robert S Crupi, MD ${ }^{\mathrm{a}}$

[15]. Journal of the American Society of Hypertension, Volume 4, Issue 2,March-April 2010, Pages 68-78,Hypertension in pregnancy .Marshall D. Lindheimer, MD, Sandra J. Taler, MD, F. Gary Cunningham, MD 